\title{
Acupuncture for heart failure: a Bayesian network systematic review and meta-analysis protocol
}

\author{
Fengya Zhu ${ }^{1} \wedge$, Shao Yin $^{2} \wedge$, Gang Zheng ${ }^{3}$, Yue Zhong ${ }^{1}$, Daohui Gan ${ }^{1}$, Lvyu Deng ${ }^{1}$, Shumin Chen ${ }^{4}$, \\ Lanying Yang ${ }^{1}$, Liuying $\mathrm{Li}^{1}$ \\ ${ }^{1}$ Traditional Chinese Medicine Department, Zigong First People's Hospital, Zigong, China; ${ }^{2}$ Clinical Medical School, Hospital of Chengdu \\ University of Traditional Chinese Medicine, Chengdu, China; ${ }^{3}$ Respiratory and Critical Care Medicine, Zigong First People's Hospital, Zigong, \\ China; ${ }^{4}$ Emergency Intensive Care Unit, Zigong First People's Hospital, Zigong, China \\ Correspondence to: Liuying Li. Traditional Chinese Medicine Department, Zigong First People's Hospital, Zigong 643000, China. \\ Email: arenally@sina.com.
}

\begin{abstract}
Background: Heart failure (HF) is the final stage of cardiovascular disease development, and clinical studies on acupuncture for the treatment of HF are increasing. However, the efficacy and safety of these acupuncture methods are unclear. The purpose of this study is to conduct a meta-analysis of the relevant randomized controlled trials (RCTs) to explain their efficacy and safety through high-quality evidence.

Methods: The databases of the Cochrane Library, PubMed, Web of Science, SinoMed, China National Knowledge Infrastructure (CNKI), Wan-fang, and VIP will be searched from inception date to June 2021. The search will not be restricted by language. This study will include RCTs of various acupuncture methods for HF. Study selection, data extraction, and research quality assessments will be conducted independently by two researchers. The primary outcome is the efficacy rate and heart function, the secondary outcomes are quality of life scores, rehospitalization, and adverse reactions/events. The software RevMan 5.3 and Aggregate Data Drug Information System (ADDIS) will be used for network meta-analysis (NMA). Risk of bias, meta-regression, subgroup analysis, and sensitivity analysis will be performed, and the quality of evidence will be assessed using the Grading of Recommendations Assessment, Development, and Evaluation (GRADE) framework.

Discussion: This study will confirm the efficacy and safety of acupuncture in improving the clinical symptoms and heart function of HF, or elucidate which method of acupuncture is better suited to this purpose.
\end{abstract}

Keywords: Acupuncture; heart failure; systematic review; protocol

Submitted Jul 22, 2021. Accepted for publication Sep 16, 2021.

doi: 10.21037/apm-21-2131

View this article at: https://dx.doi.org/10.21037/apm-21-2131

\section{Introduction}

Heart failure (HF) is the final stage of the development of a variety of cardiovascular diseases. Hypertension, cardiomyopathy, myocardial infarction, hemodynamic overload, inflammation, and other causes can induce myocardial damage and changes in myocardial structure and function. Eventually, the ventricular pumping and/or filling function is decreased. According to the American Heart Association's updated reports in 2011 and 2016, the global prevalence of $\mathrm{HF}$ has increased from 23 million to 37.7 million $(1,2)$, which is about $3-5 \%$ of the total population. The incidence of $\mathrm{HF}$ in developed countries is about $2 \%$. Data updated by the American Heart Association in 2017 show that an estimated 6.5 million

^ ORCID: Fengya Zhu, 0000-0002-2574-0748; Shao Yin, 0000-0003-3865-3122. 
Americans over the age of 20 experience HF, and medical expenses are expected to increase from US \$20.9 billion in 2012 to $\$ 53.1$ billion in 2030. In developing countries, such as Latin America and parts of Asia, the number of $\mathrm{HF}$ patients is also increasing, and with changes in lifestyle, the diseased population is becoming progressively younger. Among Chinese adults aged 35 years or older, the weighted prevalence of $\mathrm{HF}$ is $1.3 \%$, which equates to 18 million people (3). According to World Bank statistics, the annual global economic cost is 108 billion dollars (4-6). Judging from the current evidence, although the age-specific death rate has dropped by $39 \%$, the number of deaths from cardiovascular diseases worldwide has increased by $41 \%$ due to population growth, population aging, and disease epidemiological changes (7). Subsequently, HF has become the fastest growing cardiovascular disease in the world.

It is a hemodynamic disease whose pathophysiological basis is cardiac output, cardiac contractility, filling pressure, wall stress during systolic and diastolic function, and heart rhythm. The diagnosis of HF is mainly through natriuretic peptide (NP), electrocardiogram (ECG), and echocardiography. Echocardiography is the most sensitive for patients with suspected $\mathrm{HF}$, and the most specific seems to be brain natriuretic peptide (BNP) or the $\mathrm{N}$-terminal pro-B type natriuretic peptide (NT-proBNP). At present, conventional treatments for HF include diuretics, angiotensin-converting enzyme inhibitors (ACEIs), angiotensin receptor blockers (ARB), beta-receptor blockers, aldosterone antagonists, digitalis, or vasodilators, yet the rehospitalization and mortality toll of $\mathrm{HF}$ has continued to increase (8-10). Therefore, it is necessary to find complementary and alternative therapies.

Related studies have shown that many Chinese herbal decoctions or injections have been used in adjuvant treatment of HF and achieved good results, but their safety is controversial due to the uncertainty of herbal composition (11-15). Acupuncture is an important component of traditional Chinese medicine (TCM). In recent years, relevant studies have made preliminary progress in treating HF with acupuncture. The results of an randomized controlled trial (RCT) showed that 6-min walking distance (6-MWD) increased by $32 \pm 7 \mathrm{~m}$, post-exercise recovery (after maximum exercise) and carbon dioxide ventilation equivalent slope $\left(\mathrm{VE} / \mathrm{VCO}_{2}\right)$ were improved, and heart rate variability increased in the acupuncture group and decreased in the placebo acupuncture group. In the acupuncture group, "general health" and "physical pain" in the medical outcomes study Short Form 36-Item Health Survey (SF-36) were significantly improved (16,17). In 2016, a systematic review included 7 RCTs of acupuncture for $\mathrm{HF}$, but due to the poor methodology, the efficacy has not yet been concluded (18). In 2019, 32 RCTs involving 2,499 patients were reviewed, suggesting that acupuncture may be a promising intervention for the treatment of $\mathrm{HF}$ as an adjuvant therapy; however, the evidence remains inconclusive (19). The efficacy and safety of acupuncture has not been explained due to the array of acupuncture methods used. Which method of acupuncture is more effective in treating HF remains to be confirmed. Network metaanalysis (NMA) assesses both direct and indirect evidence (20-22), so we aim perform NMA to comprehensively evaluate the efficacy and safety of different acupuncture methods for HF.

This study will select RCTs of acupuncture for HF. All participants will have been diagnosed with $\mathrm{HF}$, and the treatment group will include all acupuncture methods based on acupoints, while the control group will incorporate placebo, sham-acupuncture, medication, routine care, and so on. The primary outcome will be the efficacy rate and heart function, the secondary outcomes will be quality of life scores, rehospitalization, and adverse reactions/events. The study will focus on directly or indirectly evaluating the efficacy and safety of various types of acupuncture for HF through NMA, so as to provide high-quality evidence-based medical evidence for clinical decision making. We present the following article in accordance with the PRISMA reporting checklist (available at https://dx.doi.org/10.21037/ apm-21-2131).

\section{Methods}

\section{Registration of this review}

The protocol is structured and implemented according to the Preferred Reporting Items for Systematic Reviews and Metaanalyzes Protocols (PRISMA-P) statement guidelines (23), and registered on OSF as: 10.17605/OSF.IO/EBS23 (https:// osf.io/ebs23).

\section{Etbics}

Ethics approval is not needed because the data will not contain individual participant data, and there are no concerns about privacy. 


\section{Criteria for including studies in this review}

\section{Type of study}

This study will include all RCTs of various acupuncture therapies for HF. Quasi-randomized trials, animal experiments, case reports, self-controlled trials, case studies, and conference papers will be excluded.

\section{Type of participants}

This study will include all participants diagnosed with HF, whether it is acute heart failure (AHF) or chronic heart failure (CHF), regardless of age, gender, race, educational level, source of cases, disease duration, and cause of illness. No restriction was imposed on the cause of HF, except for those cases caused by valvular heart disease, pregnancy, chemotherapy, congenital defects, or surgery.

\section{Type of interventions}

The treatment group will include all acupuncture methods based on acupoints, such as manual acupuncture, electroacupuncture, warm acupuncture, thread embedding, moxibustion, and acupoint application. Methods that are not based on acupoint therapy and other TCM methods will be excluded, such as acupoint injection (because such injections contain some drug ingredients), ear acupuncture, scalp acupuncture, fire needle, transcutaneous electric nerve stimulation (which focuses on the site, not the acupoint), Chinese herbal medicine, massage, tuina, or qigong.

\section{Type of controls}

The control group will include placebo, sham-acupuncture (a method that does not pierce the patient's skin or does not reach the depth of acupuncture. It may be meridian or nonmeridian and non-acupoint), medication, routine care, and so on. If the treatment group adopts acupuncture combined with other treatment methods, the control group should adopt the same treatment methods without acupuncture.

In addition, RCTs that compare different kinds of acupuncture will be excluded.

\section{Type of outcomes}

Studies reporting 1 or more of the following outcome indicators will be included. The time point of assessing outcomes is after the end of treatment. The main outcomes include the effective rate and heart function [left ventricular ejection fraction (LVEF), left ventricular end-diastolic dimension (LVEDD), left ventricular end-systolic dimension (LVESD), cardiac index (CI), cardiac output (CO), stroke volume (SV), 6-min walk test (6-MWT), BNP, NT-proBNP]. The secondary outcomes include quality of life scores [SF-36, the EuroQol-5 Dimension (EQ-5D), the Minnesota Living with Heart Failure Questionnaire (MLHFQ), the Kansas City Cardiomyopathy Questionnaire (KCCQ) (24)], rehospitalization, and adverse reactions/events.

\section{Data sources and search methods}

\section{Search strategy}

We will electronically and manually retrieve all relevant RCTs from inception date to June 2021, without language and publication restrictions. Databases including the Cochrane Library, PubMed, Web of Science, SinoMed, China National Knowledge Infrastructure (CNKI), Wan-fang, and VIP will be searched. The following search terms will be used: "heart failure", "cardiac failure", "myocardial failure", "heart insufficiency", "cardiac insufficiency", "ventricular dysfunction", "cardiomyopathies", "acupuncture", "manual acupuncture", "electro-acupuncture", "warm acupuncture", "thread embedding", "moxibustion", "acupoint application", "randomized controlled trial", "randomized controlled", "controlled clinical trial", "clinical trial". The search strategy for the PubMed database is shown in Table 1. Corresponding adjustments according to the search terms will be made when searching other databases. In addition, we will manually search the references for all relevant articles to identify other eligible studies.

\section{Data collection and analysis}

\section{Selection of studies}

All reviewers have completed strict training and two reviewers (Shumin Chen and Lanying Yang) will screen all eligible studies independently based on the titles and abstracts. Then, the search results will be imported into EndNote software (V.X9, https://endnote.com/) to eliminate duplicate documents. The flow process of study selection is shown in Figure 1. When differences occur, the two reviewers will discuss to reach consensus, and any unresolved problems will be decided by the third reviewer (Liuying Li).

\section{Data extraction and analysis}

Full texts will be read and data extracted based on the Excel program (https://office.microsift.com/excel) by Daohui Gan and Lvyu Deng. Extracted data will include the following items: (I) general information: title, country, language, first author, contact information, publication year, funding, trial 
Table 1 Search strategy in PubMed database

\begin{tabular}{|c|c|}
\hline Number & Search items \\
\hline 1 & Heart failure (MeSH Terms) \\
\hline 2 & Cardiac failure (all fields) \\
\hline 3 & Myocardial failure (all fields) \\
\hline 4 & Heart insufficiency (all fields) \\
\hline 5 & Cardia insufficiency (all fields) \\
\hline 6 & Ventricular dysfunction (all fields) \\
\hline 7 & Cardiomyopathies (all fields) \\
\hline 8 & 1 or $2-7$ \\
\hline 9 & Acupuncture (MeSH Terms) \\
\hline 10 & Manual acupuncture (all fields) \\
\hline 11 & Electro-acupuncture (all fields) \\
\hline 12 & Warm acupuncture (all fields) \\
\hline 13 & Thread embedding (all fields) \\
\hline 14 & Moxibustion (all fields) \\
\hline 15 & Acupoint application (all fields) \\
\hline 16 & 9 or $10-15$ \\
\hline 17 & Randomized controlled trial (MeSH Terms) \\
\hline 18 & Randomized controlled (all fields) \\
\hline 19 & Controlled clinical trial (all fields) \\
\hline 20 & Clinical trial (all fields) \\
\hline 21 & 17 or $18-20$ \\
\hline 22 & 8 and 16 and 21 \\
\hline
\end{tabular}

registration, conflicts of interest, ethical perception; (II) research design: participants, sample size, randomization, allocation concealment, blinding; (III) interventions: according to the Standards for Reporting Interventions in Clinical Trials of Acupuncture (STRICTA) standard, the features of acupuncture will be reported in detail. Control intervention (placebo, sham-acupuncture, medication, or routine care) also will be reported, in particular, the name, dosage, frequency, course of medicine; (IV) research outcomes: outcome assessment time point, primary outcome, secondary outcome, adverse reactions/events, and conclusions. Any differences will be resolved by discussion between the two reviewers, and any further disagreement will be resolved by the third author (Liuying Li).

\section{Assessment of risk of bias}

The Cochrane Collaboration's tool to assess the risk of bias tool (ROB) will be used by two reviewers (Gang Zheng and Yue Zhong) for each study (25). The evaluation includes random sequence generation, allocation concealment, blinding of participants and assessors, blinding of outcome assessment, incomplete outcome data, selective reporting, and other bias. The assessment results will be classified into three levels: low risk, high risk, and unclear risk. Any disagreements will be arbitrated by the third reviewer (Liuying Li).

\section{Data synthesis}

We will use the software RevMan (version 5.3, Copenhagen: The Nordic Cochrane Center, The Cochrane Collaboration, 2014) to conduct a pairwise meta-analysis of the direct comparison results obtained from the literature. Continuous data will be analyzed using mean difference (MD) or standard mean difference (SMD) with $95 \%$ confidence intervals (CI), risk ratio (RR), or odds ratio (OR) with $95 \%$ CI for dichotomous data. For the results of indirect comparison, we will use the Aggregate Data Drug Information System (ADDIS) to perform NMA on the random effects model. Comprehensive analysis of all direct and indirect results will be conducted based on the Bayesian framework and Markov Chain Monte Carlo (MCMC) method. The surface under the cumulative ranking curve analysis (SUCRA) by Stata software (StataCorp. LLC, College Station, TX, USA) will visually display the ranking of each acupuncture method. If necessary, a narrative description of the results will be provided.

\section{Management of missing data}

We will attempt to contact the first author or corresponding author of the original article to obtain missing or insufficient data. If it is not available, we will analyze the existing data. Sensitivity analysis will be conducted to assess the potential impact of missing data on the results.

\section{Assessment of beterogeneity}

The heterogeneity of the included trials will be assessed by the $\mathrm{I}^{2}$ test in a forest plot. If $\mathrm{P}>0.05$ and $\mathrm{I}^{2}<50 \%$, it will be considered that there is no great heterogeneity 


\section{Identification of studies via databases}

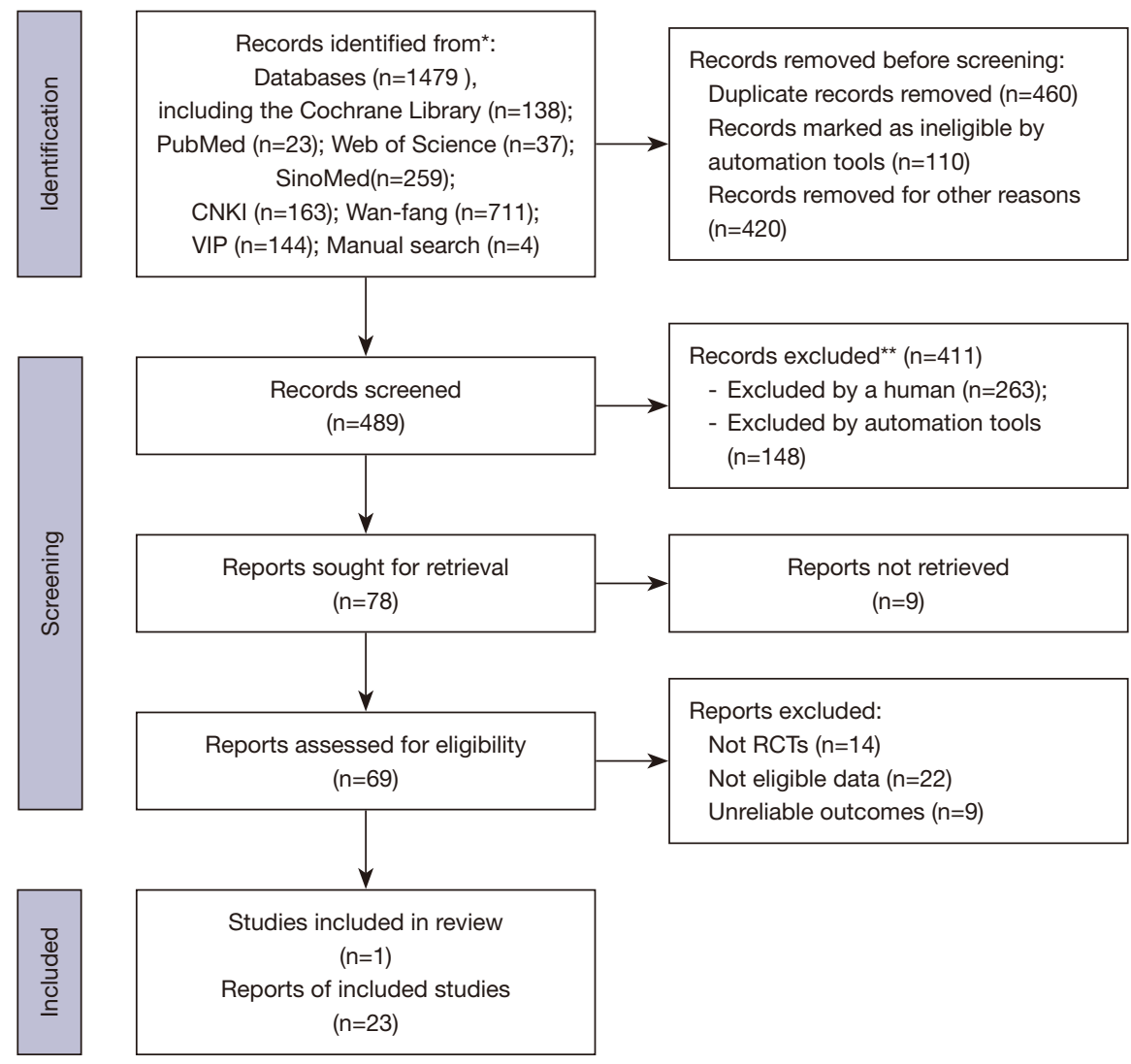

*, consider, if feasible to do so, reporting the number of records identified from each database or register searched (rather than the total number across all tabases/registers). **, if automation tools were used, indicate how many records were excluded by a human and how many were excluded by automation tools.

Figure 1 PRISMA flow diagram of the study selection process.

in the result, the fixed-effects model will be adopted. If $\mathrm{P}<0.05$ and $\mathrm{I}^{2}>50 \%$, the result will be viewed as having significant heterogeneity, and the random-effects model will be utilized. In addition, If the heterogeneity is large, meta-analysis will not be performed, and the source of heterogeneity will be explored. If necessary, subgroup analysis will also be considered.

\section{Assessment of reporting bias}

If the number of the studies included exceeds 10 trials, we will use a funnel plot to assess reporting biases. Significant publication bias will be considered at $\mathrm{P}<0.05$.

\section{Subgroup analysis}

If the studies have high heterogeneity, subgroup analysis will be performed based on type of participants (AHF or $\mathrm{CHF}$ ), different acupuncture methods, treatment courses, different types of control group, and clinical differences to investigate the source of heterogeneity.

\section{Sensitivity analysis}

Sensitivity analysis will be used to verify the reliability of results. According to the Cochrane Handbook, sensitivity analysis will be conducted in the three aspects of methodological quality, sample size, and statistical model. 
We will exclude studies with poor research quality, small sample size, and high risk of bias. Types of acupuncture, choice of acupoints, frequency, course, and missing data will also be taken into account.

\section{Grading of quality of evidence}

According to the risk of bias, indirectness, inconsistency, imprecision, and publication bias, two reviewers (Fengya Zhu and Shao Yin) will independently evaluate the quality of the evidence using the Grading of Recommendations Assessment, Development, and Evaluation System (GRADE) (26). The assessment is divided into four levels: high, moderate, low, and very low. Any disagreements will be arbitrated by the third reviewer (Liuying $\mathrm{Li}$ ).

\section{Discussion}

\section{The potential mechanism of acupuncture for $\mathrm{HF}$}

Many studies have explored the mechanism of acupuncture for HF, involving multiple systems and fields. Neuroendocrine-immune networks and inflammatory mediators play an important role in the development of HF (27). Researchers have earlier shown that acupuncture can inhibit sympathetic nerve activity in patients with advanced $\mathrm{HF}$ and improve prognosis in patients (28). Meanwhile, electroacupuncture can improve the cardiac function and ventricular remodeling of rats with CHF by inhibiting sympathetic nerve excitation, and reduce the infarct size (29). In the state of CHF, electrical stimulation of the vagus nerve can stimulate the immune system in the spleen and inhibit the synthesis of TNF synthesis and inflammation (30). In addition, acupuncture can also regulate the renin-angiotensin-aldosterone system (RAAS) inhibiting myocardial fibrosis in CHF, and reduce serum BNP, REN, ALD, NE and Ang II levels (31,32). However, the pathogenesis of HF is still unclear, and the mechanism of acupuncture can only be studied on the basis of existing evidence.

\section{The importance of this review}

The common cardiovascular disease, HF, has very high rates of prevalence, rehospitalization, and mortality, which seriously affects the quality of life of patients. Due to high medical costs, it brings a heavy burden to the family and society of HF patients. The goals of treatment for HF are to improve the efficacy, heart function, and quality of life, and reduce rehospitalization and mortality. In recent years, many clinical studies on HF treated by acupuncture have been published, and good progress has been made. However, there are many methods of acupuncture, and its efficacy and safety of have not yet been determined. Therefore, for the first time, we will comprehensively evaluate the efficacy and safety of various kinds of acupuncture for HF by conducting NMA, and potentially ascertain which method of acupuncture is most suited to this purpose.

\section{Selection of indicators}

The efficacy rate is the most direct and basic outcome. Heart function is an objective index reflecting whether acupuncture is effective in treating patients with HF. The SF-36 has been widely used in the field of general population quality of life measurement, clinical trial effect evaluation, and health policy evaluation. The EQ-5D is widely used worldwide as a multidimensional healthrelated quality of life measurement (27). The KCCQ and MLHFQ are 2 of the most widely used and validated questionnaires for assessing the specific quality of life for HF (28). In this study, general health questionnaires and HF specific questionnaires were selected in order to obtain comprehensive research results. Rehospitalization is a common endpoint in HF clinical trials, and it is necessary to conduct a meta-analysis as a secondary outcome. Therefore, the efficacy rate and heart function were selected as primary outcomes in this study, and quality of life scores (SF-36, EQ-5D, MLHFQ, KCCQ), rehospitalization, and adverse reactions/events were selected as secondary outcomes.

\section{Conduction of a meta-analysis}

This study will be the first to perform a Bayesian NMA of acupuncture for HF and follow the "Methods" section for each of the tasks in turn. Then, the MCMC method will be used to synthesize all direct and indirect results, and the ranking of various acupuncture methods will be presented through SUCRA. We will rigorously assess the risk of bias using the Cochrane assessment tool, with GRADE assessing the quality of evidence. The aim is to provide evidencebased medical documentation for the efficacy and safety of acupuncture for HF, so as to optimize the clinical practice of acupuncture and further research. 


\section{Strengths and limitations of this review}

To our knowledge, this study will be first to directly or indirectly compare the efficacy and safety of various acupuncture methods for HF using Bayesian NMA. At the same time, we will strictly adhere to the PRISMA-P statement guideline and the quality of evidence will be assessed by GRADE. However, we must acknowledge that different types of acupuncture and quality of the original study may increase the risk of heterogeneity.

\section{Acknowledgments}

We would like to thank Dr. Stephen for his assistance in improving this manuscript.

Funding: This work was funded by Sichuan Medical Association (2018SHD2-3), Science and Technology Department of Zigong City (2019IZSF43), the Key Discipline Construction Project of Sichuan Administration of Traditional Chinese Medicine (No. 202072). The sponsor is not involved in this study and the authors do not have any conflicts of interest to declare.

\section{Footnote}

Reporting Checklist: The authors have completed the PRISMA reporting checklist. Available at https://dx.doi. org/10.21037/apm-21-2131

Conflicts of Interest: All authors have completed the ICMJE uniform disclosure form (available at https:// dx.doi.org/10.21037/apm-21-2131). All authors reported that the expenditures related to this research were funded by the Key Discipline Construction Project of Sichuan Administration of Traditional Chinese Medicine (202072), Sichuan Medical Association (2018SHD2-3) and Science and Technology Department of Zigong City (2019IZSF43). The authors have no other conflicts of interest to declare.

Ethical Statement: The authors are accountable for all aspects of the work in ensuring that questions related to the accuracy or integrity of any part of the work are appropriately investigated and resolved. Ethics approval is not needed because the data will not contain individual participant data, and there are no concerns about privacy.

Open Access Statement: This is an Open Access article distributed in accordance with the Creative Commons Attribution-NonCommercial-NoDerivs 4.0 International License (CC BY-NC-ND 4.0), which permits the noncommercial replication and distribution of the article with the strict proviso that no changes or edits are made and the original work is properly cited (including links to both the formal publication through the relevant DOI and the license). See: https://creativecommons.org/licenses/by-nc-nd/4.0/.

\section{References}

1. Bui AL, Horwich TB, Fonarow GC. Epidemiology and risk profile of heart failure. Nat Rev Cardiol 2011;8:30-41.

2. Ziaeian B, Fonarow GC. Epidemiology and aetiology of heart failure. Nat Rev Cardiol 2016;13:368-78.

3. Hao G, Wang X, Chen Z, et al. Prevalence of heart failure and left ventricular dysfunction in China: the China Hypertension Survey, 2012-2015. Eur J Heart Fail 2019;21:1329-37.

4. Ponikowski P, Anker SD, AlHabib KF, et al. Heart failure: preventing disease and death worldwide. ESC Heart Fail 2014;1:4-25.

5. Benjamin EJ, Blaha MJ, Chiuve SE, et al. Heart Disease and Stroke Statistics-2017 Update: A Report From the American Heart Association. Circulation 2017;135:e146-603.

6. Cook C, Cole G, Asaria P, et al. The annual global economic burden of heart failure. Int J Cardiol 2014;171:368-76.

7. Roth GA, Forouzanfar MH, Moran AE, et al. Demographic and epidemiologic drivers of global cardiovascular mortality. N Engl J Med 2015;372:1333-41.

8. Špinar J, Špinarová L, Vítovec J. Pathophysiology, causes and epidemiology of chronic heart failure. Vnitr Lek 2018;64:834-8.

9. Ponikowski P, Voors AA, Anker SD, et al. 2016 ESC Guidelines for the diagnosis and treatment of acute and chronic heart failure: The Task Force for the diagnosis and treatment of acute and chronic heart failure of the European Society of Cardiology (ESC). Developed with the special contribution of the Heart Failure Association (HFA) of the ESC. Eur J Heart Fail 2016;18:891-975.

10. Yancy CW, Jessup M, Bozkurt B, et al. 2017 ACC/ AHA/HFSA Focused Update of the 2013 ACCF/AHA Guideline for the Management of Heart Failure: A Report of the American College of Cardiology/American Heart Association Task Force on Clinical Practice Guidelines and the Heart Failure Society of America. Circulation 2017;136:e137-61. 
11. Wang J, Yang R, Zhang F, et al. The Effect of Chinese Herbal Medicine on Quality of Life and Exercise Tolerance in Heart Failure With Preserved Ejection Fraction: A Systematic Review and Meta-Analysis of Randomized Controlled Trials. Front Physiol 2018;9:1420.

12. Wang Y, Wang Q, Li C, et al. A Review of Chinese Herbal Medicine for the Treatment of Chronic Heart Failure. Curr Pharm Des 2017;23:5115-24.

13. Mei J, Xu H, Xu FQ, et al. Oral Chinese Herbal Medicine for Heart Failure with Preserved Ejection Fraction: A Meta-Analysis. Chin J Integr Med 2019;25:770-7.

14. Bai D, Yue GX, Wang RH, et al. Clinical characteristics of five traditional Chinese medicine injections in treating heart failure based on Meta-analysis literature. Zhongguo Zhong Yao Za Zhi 2018;43:4152-62.

15. Wen-Ting S, Fa-Feng C, Li X, et al. Chinese medicine shenfu injection for heart failure: a systematic review and meta-analysis. Evid Based Complement Alternat Med 2012;2012:713149.

16. Ware JE Jr, Gandek B. Overview of the SF-36 Health Survey and the International Quality of Life Assessment (IQOLA) Project. J Clin Epidemiol 1998;51:903-12.

17. Kristen AV, Schuhmacher B, Strych K, et al. Acupuncture improves exercise tolerance of patients with heart failure: a placebo-controlled pilot study. Heart 2010;96:1396-400.

18. Lee H, Kim TH, Leem J. Acupuncture for heart failure: A systematic review of clinical studies. Int J Cardiol 2016;222:321-31.

19. Liang B, Yan C, Zhang L, et al. The Effect of Acupuncture and Moxibustion on Heart Function in Heart Failure Patients: A Systematic Review and Meta-Analysis. Evid Based Complement Alternat Med 2019;2019:6074967.

20. Salanti G, Del Giovane C, Chaimani A, et al. Evaluating the quality of evidence from a network meta-analysis. PLoS One 2014;9:e99682.

21. Caldwell DM, Ades AE, Higgins JP. Simultaneous comparison of multiple treatments: combining direct and indirect evidence. BMJ 2005;331:897-900.

22. Chaimani A, Higgins JP, Mavridis D, et al. Graphical

Cite this article as: Zhu F, Yin S, Zheng G, Zhong Y, Gan D, Deng L, Chen S, Yang L, Li L. Acupuncture for heart failure: a Bayesian network systematic review and meta-analysis protocol. Ann Palliat Med 2021;10(10):11148-11155. doi: 10.21037/apm-212131 tools for network meta-analysis in STATA. PLoS One 2013;8:e76654.

23. Moher D, Shamseer L, Clarke M, et al. Preferred reporting items for systematic review and meta-analysis protocols (PRISMA-P) 2015 statement. Syst Rev 2015;4:1.

24. Rector TS, Cohn JN. Assessment of patient outcome with the Minnesota Living with Heart Failure questionnaire: reliability and validity during a randomized, double-blind, placebo-controlled trial of pimobendan. Pimobendan Multicenter Research Group. Am Heart J 1992;124:1017-25.

25. Higgins JP, Altman DG, Gøtzsche PC, et al. The Cochrane Collaboration's tool for assessing risk of bias in randomised trials. BMJ 2011;343:d5928.

26. Schünemann HJ, Oxman AD, Brozek J, et al. Grading quality of evidence and strength of recommendations for diagnostic tests and strategies. BMJ 2008;336:1106-10.

27. Balestroni G, Bertolotti G. EuroQol-5D (EQ-5D): an instrument for measuring quality of life. Monaldi Arch Chest Dis 2012;78:155-9.

28. Yee D, Novak E, Platts A, et al. Comparison of the Kansas City Cardiomyopathy Questionnaire and Minnesota Living With Heart Failure Questionnaire in Predicting Heart Failure Outcomes. Am J Cardiol 2019;123:807-12.

29. Ma L, Cui B, Shao Y, et al. Electroacupuncture improves cardiac function and remodeling by inhibition of sympathoexcitation in chronic heart failure rats. Am J Physiol Heart Circ Physiol 2014;306:H1464-H1471.

30. Fox D. The shock tactics set to shake up immunology. Nature 2017;545:20-2. Erratum in: Nature 2017;545:398.

31. Gou D. The research of acupuncture therapy on rabbits with chronic heart failure. Southwest Medical University, 2017.

32. Liu HWS. Effect of acupuncture on myocardial fibrosis in rats with chronic heart failure. Southwest Medical University, 2020.

(English Language Editor: J. Jones) 